
\title{
Nomograms to predict prognosis of hepatocelluar carcinoma without major vascular invasion after primary liver transplantation
}

\author{
Junyi Shen ${ }^{1, *}$, Chuan Li ${ }^{1, *}$, Jun Wen ${ }^{2}$, Tianfu Wen ${ }^{1}$, Lvnan Yan ${ }^{1}$, Bo Li $^{1}$, Jiayin Yang ${ }^{1}$ \\ and Highness Tholakkara Nazar ${ }^{1}$ \\ ${ }^{1}$ Department of Liver Surgery and Liver Transplantation Center, West China Hospital, Chengdu, Sichuan Province, China \\ ${ }^{2}$ Department of General Surgery, The Third People's Hospital of Chengdu, Chengdu, Sichuan Province, China \\ "These authors contributed equally to this work \\ Correspondence to: Tianfu Wen, email: cdwentianfu@126.com \\ Keywords: nomogram; prognosis; hepatocelluar carcinoma; liver transplantation \\ Received: May 23, $2017 \quad$ Accepted: January 02, $2018 \quad$ Published: January 02, 2018 \\ Copyright: Shen et al. This is an open-access article distributed under the terms of the Creative Commons Attribution License 3.0 \\ (CC BY 3.0), which permits unrestricted use, distribution, and reproduction in any medium, provided the original author and source \\ are credited.
}

\section{ABSTRACT}

Background: A model to predict individual prognosis after liver transplantation (LT) has not been clearly established. We aimed to create nomograms for prediction of individual survival after LT for hepatocelluar carcinoma (HCC).

Results: There were $128(61.2 \%)$ patients within the Milan criteria. Before transplantation, $43(20.6 \%)$ patients received transcatheter arterial chemoembolization (TACE) and $22(10.5 \%)$ received radiofrequency ablation(RFA). Multivariate predictors of survival included tumor size, tumor number and tumor differentiation. Nomograms for OS and recurrence free survival (RFS) have been created. Discrimination and calibration of the nomograms revealed good predictive abilities(C-index, RFS: 0.74; OS: 0.70). Compared with Milan criteria, UCSF criteria, up-to-seven criteria and Hangzhou criteria, the OS nomogram improved accuracy in predicting prognosis after transplantation for HCC, $(p<0.05$ for all). Finally, three subgroups were generated based on the total risk points ( $A^{\prime}$ : 1.88-76.5 points; $B^{\prime}$ : 76.6-113.9points; $C^{\prime}$ : 113.9-151.3points). The 5-year survival rate were $86.1 \%$ for group $A^{\prime}, 59.1 \%$ for group $B^{\prime}$, and $28.9 \%$ for group $C^{\prime}$, respectively.

Conclusions: The nomograms had good ability in predicting prognostic survival for HCC patients after LT. Patients with score above 113.9 points had poor survival after LT.

Materials and method: Between Juanuary 2002 and September 2015, 209 HCC patients who received liver transplantation in the West China Hospital were collected for this study. Prognostic nomograms predicting post-transplant prognosis were developed from a multivariate cox regression. The prediction power of the nomograms was tested by C-statistic and calibration plots.

\section{INTRODUCTION}

Hepatocellular carcinoma (HCC) is the third most common cause of cancer-related death in the AsiaPacific region. In particular, China accounts for about $50 \%$ of all HCC cases worldwide because of high prevalence of hepatitis B viral (HBV) infection [1]. Liver transplantation (LT) is a rational therapeutic option for HCC patients because it addresses the cancer and also treats the underlying liver disease. Considering the shortage of liver donor and long-term survival, in 1996, Mazzaferro, et al. proposed the widely accepted liver transplantation criteria-the Milan criteria (Single tumor $\leq 5 \mathrm{~cm}$ in size or $\leq 3$ tumors each $\leq 3 \mathrm{~cm}$ in size, and no macrovascular invasion) [2]. Patients within the Milan criteria could achieve 5-year survival of above $70 \%$ [3]. Recently, expanded criteria had been proposed, such as UCSF criteria, up-to-seven criteria and Hangzhou 
criteria [4-6]. These criterias enabled more patients to qualify as transplant candidates but without greatly compromising the long-term survival. According the previous studies, the 5-year survival could reach up to $78.3 \%$ for the Milan criteria, $72.4 \%$ for the UCSF, $71.2 \%$ for the up-to-seven and $72.3 \%$ for the Hangzhou criteria [4-6]. Recently, large sample size studies from eastern and western liver transplantation centers had reported the superiority of transplantation over resection for HCC beyond the Milan criteria without major vascular invasion [3, 7]. Moreover, study from Bruix, et al. and Volk et al. suggested that transplantation for HCC without dropping to $50 \%$ of 5 -year overall survival was acceptable $[8,9]$. These evidences suggested that some HCC patients beyond the transplantation criterias could benefit from the transplantation. However, when expanding the transplantation criteria, we still observed that there was a decrease in the long-term survival. This suggested that tumor characteristics like tumor number and tumor size remained to be determinant factors for the prognosis $[10,11]$. Since more HCC patient receiving the LT, the individual survival prediction was crucial in clinical practice. An accurate prognostic prediction after transplantation could guide postoperative monitoring and adjuvant therapy. Currently, there was no standard approach for prediction of prognosis for transplantation patients. Numerous studies had suggested that nomogram is of great value to predict long-term prognosis using a simple graphical representation in various cancers including HCC [12-14].

The aim of the current study was to create nomograms to predict OS and RFS following LT and stratified the prognosis.

\section{RESULTS}

\section{Demographics and clinicopathological characteristics}

A total of 209 HCC patients were included in the present study. Baseline information was summarized in Table 1. There were 183 patients with age $\leq 60 \mathrm{y}$ $(87.6 \%)$ and 184 male patients $(88.0 \%)$. The majority of patients $(92.3 \%)$ suffered from HBV infection. Before transplantation, $43(20.6 \%)$ had underwent TACE and $22(10.5 \%)$ patients had underwent RFA. There were 58 patients $(27.8 \%)$ with the presence of microvascular invasion (MVI), 16 patients $(7.7 \%)$ with the presence of satellite lesions, 71 patients $(34.0 \%)$ with poor tumor differentiation. 39 patients $(18.7 \%)$ had two tumors and $27(12.9 \%)$ had more tumors. Tumor size was 4.0 $\pm 3.0 \mathrm{~cm}$. Among the patients, there were 128 patients (61.2\%) within the Milan criteria, 147 patients $(70.3 \%)$ within UCSF criteria, 146 patients (69.9\%) within up-toseven criteria and 156 patients (74.6\%) within Hangzhou criteria. (Table 1).

\section{Risk factors analysis and nomograms construction}

The median follow-up time was 48.6 months (range, 1 to 174.0 moths). During this period, 22 patients (10.5\%) suffered from HCC recurrence, and 51 patients $(24.4 \%)$ died. .Overall survival (OS) was $83.4 \%, 75.1 \%, 73.3 \%$ at $1-, 3-$, and 5-years, respectively. In the univariate analysis, tumor size, tumor number, tumor differentiation and meld score with $p<0.1$ entered into multivariate analysis. Ultimately, tumor size ( $p=0.001$, hazard ratio (HR) 1.129, 95\%confidence interval (CI) 1.050-1.213), tumor number $(p=0.036$, HR $1.148,95 \%$ CI $1.023-1.996)$ and tumor differentiation $(p<0.001$, HR 3.015, 95\% CI 1.718-5.291) remained to be significant in multivariate analysis.

Similarly, the univariate analysis suggested that tumor size, tumor number, tumor differentiation, satellite lesions and Child-Pugh score were significantly associated with RFS, while the multivariate analysis showed that tumor number $(p=0.015$, HR $1.903,95 \%$ CI 1.132 $3.199)$, tumor differentiation ( $p=0.018$, HR $2.802,95 \%$ CI 1.193-6.580) and satellites ( $p=0.002$, HR 4.608, 95\% CI 1.760-12.068) were independently associated with RFS of HCC patients after liver transplantation (Table 2).

The nomogram predicting 1-, 3- and 5-year OS were constructed based on the independently prognostic markers for prognosis in Cox model. The risk of tumor size on prognosis was linear and tumor size $=16 \mathrm{~cm}$ could be assigned 100 points. Tumor number (one /two/ more) was assigned with $0 / 18.3 / 36.7$ points for OS and 0/48.2/96.4 points for RFS, respectively. Poor tumor differentiation was assigned with score of 57.7 points for OS and 73.5 points for RFS, respectively. The nomogram could assign the probability of OS by summing up the scores identified on the points scale for each risk factor. The total score projected to the bottom scale indicated the probability of 1-, 3- and 5-year OS (Figure 1A). Similarly, the RFS nomogram was constructed based on the three factors (Figure 1B). A higher score was associated with worse prognosis.

\section{Predictive performance and prognostic discrimination of the nomogram}

The OS nomogram achieved a C-index of 0.70 (95\% CI: 0.62-0.78). The C-indexes of the four liver transplantation criteria in predicting OS were 0.62 for the Milan criteria, 0.61 for the UCSF, 0.59 for the up-toseven criteria, 0.61 for Hangzhou criteria, lower than the proposed nomogram (c index:0,70, $p<0.05$ for all). The bootstrap-corrected calibration curves of the nomograms for the predictive probability after surgery fitted well with the observed probability of OS at 3 or 5 years (Figure 2). The RFS nomogram achieved a C-index of $0.74(95 \%$ CI: $0.62-0.86)$. The prognosis was also analyzed by the Milan criteria, UCSF criteria, up-to -seven criteria and 
Table 1: Baseline characteristics of 209 transplantation patients

\begin{tabular}{|c|c|c|}
\hline Variables & & Number (percent) \\
\hline \multirow[t]{2}{*}{ Age (y) } & $>60$ & $26(12.4)$ \\
\hline & $\leq 60$ & $183(87.6)$ \\
\hline \multirow[t]{2}{*}{ gender } & male & $184(88.0)$ \\
\hline & female & $25(12.0)$ \\
\hline \multirow[t]{3}{*}{ preoperative therapy } & none & 144 (68.9) \\
\hline & TACE & $43(20.6)$ \\
\hline & RFA & $22(10.5)$ \\
\hline \multirow[t]{3}{*}{ etiology } & none & $14(6.7)$ \\
\hline & HBV & $193(92.3)$ \\
\hline & $\mathrm{HCV}$ & $2(1.0)$ \\
\hline \multirow[t]{2}{*}{$\mathrm{AFP}(\mathrm{ng} / \mathrm{ml})$} & $>400$ & $82(39.2)$ \\
\hline & $\leq 400$ & $127(60.8)$ \\
\hline tumor size & $\mathrm{cm}$ & $4.6 \pm 3.0$ \\
\hline \multirow[t]{3}{*}{ tumor number } & one & $143(68.4)$ \\
\hline & two & $39(18.7)$ \\
\hline & more & $27(12.9)$ \\
\hline \multirow[t]{2}{*}{ tumor differentiation } & poor & $71(34.0)$ \\
\hline & well-moderate & $138(66.0)$ \\
\hline \multirow[t]{2}{*}{ MVI } & negative & $151(72.2)$ \\
\hline & positive & $58(27.8)$ \\
\hline \multirow[t]{2}{*}{ satellites } & negative & $193(92.3)$ \\
\hline & positive & $16(7.7)$ \\
\hline \multirow[t]{2}{*}{ liver cirrhosis } & negative & $22(10.5)$ \\
\hline & positive & $187(89.5)$ \\
\hline \multirow[t]{3}{*}{ Child-Pugh } & A & $93(44.5)$ \\
\hline & B & $89(42.6)$ \\
\hline & $\mathrm{C}$ & $27(12.9)$ \\
\hline \multirow[t]{2}{*}{ Milan criteria } & within & $128(61.2)$ \\
\hline & beyond & $81(38.8)$ \\
\hline \multirow[t]{2}{*}{ UCSF criteria } & within & $147(70.3)$ \\
\hline & beyond & $62(29.7)$ \\
\hline \multirow[t]{2}{*}{ Up to Seven criteria } & within & $146(69.9)$ \\
\hline & beyond & $63(30.1)$ \\
\hline \multirow[t]{2}{*}{ Hangzhou criteria } & within & $156(74.6)$ \\
\hline & beyond & $53(25.4)$ \\
\hline Lg10 (TBIL (mmol/L)) & & $1.4 \pm 0.3$ \\
\hline $\operatorname{ALB}(g / L)$ & & $37.3 \pm 6.9$ \\
\hline Lg10 (AST(U/L)) & & $1.7 \pm 0.3$ \\
\hline $\operatorname{PLT}\left({ }^{*} 10^{9} / \mathrm{L}\right)$ & & $106.4 \pm 76.3$ \\
\hline WBC $\left({ }^{*} 10^{9} / \mathrm{L}\right)$ & & $5.6 \pm 3.1$ \\
\hline MELD score & & $11.5 \pm 4.6$ \\
\hline \multirow[t]{5}{*}{ Recurrence treatment } & resection & $1(0.5)$ \\
\hline & systematic therapy & $5(2.4)$ \\
\hline & RFA & $2(1.0)$ \\
\hline & TACE & $8(3.8)$ \\
\hline & Best care support & $6(2.9)$ \\
\hline
\end{tabular}

HBV: hepatitis B viral; HCV: hepatitis C viral; AFP: alpha-fetoprotein; MVI : microvascular invasion; TBIL: total bilirubin; AST: aspartate aminotransferase; PLT: platelete; WBC: white blood cell count; MELD: Model of End-Stage Liver Disease Score RFA: radiofrequency ablation; TACE: transarterial chemoembolization. 
Table 2: Analysis of variables associated with overall survival (OS) and recurrence free survival (RFS)

\begin{tabular}{|c|c|c|c|c|c|c|}
\hline \multirow{2}{*}{$\frac{\text { OS }}{\text { Variables }}$} & \multirow[b]{2}{*}{$p$ value } & \multicolumn{2}{|c|}{ Univarate analysis } & \multicolumn{3}{|c|}{ Multivariate analysis } \\
\hline & & HR & $95 \% \mathrm{CI}$ & $p$ value & HR & $95 \% \mathrm{CI}$ \\
\hline Age (>60 vs. $\leq 60 y)$ & 0.451 & & & & & \\
\hline gender & 0.304 & & & & & \\
\hline preoperative therapy & 0.887 & & & & & \\
\hline etiology & 0.211 & & & & & \\
\hline (HBV vs. none) & 0.934 & & & & & \\
\hline (HCV vs. none) & 0.939 & & & & & \\
\hline $\operatorname{AFP}(>400 \mathrm{vs} . \leq 400 \mathrm{ng} / \mathrm{ml})$ & 0.887 & & & & & \\
\hline tumor size & $<0.001$ & 1.147 & $1.070-1.230$ & 0.001 & 1.129 & $1.050-1.213$ \\
\hline tumor number & 0.008 & 1.578 & $1.129-2.205$ & 0.036 & 1.418 & $1.023-1.966$ \\
\hline $\begin{array}{l}\text { tumor differentiation(poor vs. } \\
\text { moderate-well) }\end{array}$ & $<0.001$ & 3.407 & $1.946-5.963$ & $<0.001$ & 3.015 & $1.718-5.291$ \\
\hline MVI & 0.452 & & & & & \\
\hline satellite lesions & 0.183 & & & & & \\
\hline liver cirrhosis & 0.561 & & & & & \\
\hline Meld score & 0.094 & & & & & \\
\hline Child-Pugh & 0.114 & & & & & \\
\hline Lg10(TBIL) & 0.335 & & & & & \\
\hline ALB & 0.821 & & & & & \\
\hline Lg10(AST) & 0.753 & & & & & \\
\hline PLT & 0.184 & & & & & \\
\hline WBC & 0.894 & & & & & \\
\hline \multicolumn{7}{|l|}{ RFS } \\
\hline Variables & $p$ value & HR & $95 \% \mathrm{CI}$ & $p$ value & HR & $95 \% \mathrm{CI}$ \\
\hline tumor size & 0.031 & 1.129 & $1.011-1.260$ & & & \\
\hline tumor number & 0.001 & 2.344 & $1.434-3.833$ & 0.015 & 1.903 & $1.132-3.199$ \\
\hline $\begin{array}{l}\text { tumor differentiation(poor vs. } \\
\text { moderate-well) }\end{array}$ & 0.006 & 3.284 & $1.425-7.621$ & 0.018 & 2.802 & $1.193-6.580$ \\
\hline satellite lesions & $<0.001$ & 7.368 & $2.980-18.217$ & 0.002 & 4.608 & $1.760-12.068$ \\
\hline Child-Pugh & 0.016 & 0.352 & $0.151-0.820$ & & & \\
\hline
\end{tabular}

HR: hazard ratio; CI confidence interval; HBV: hepatitis B viral; HCV: hepatitis C viral; AFP: alpha-fetoprotein; MVI: microvascular invasion; TBIL: total bilirubin; AST: aspartate aminotransferase; PLT: platelete; WBC: white blood cell count; MELD: Model of End-Stage Liver Disease Score.

Hangzhou criteria. Patients within these criteria had significantly better prognosis than that beyond these criteria (Figure 3).

Of all patients, total point ranged from 0 to 151 points, which was equally divided in four groups. Prognostic discrimination was performed using Kaplan-Meier method among the four groups (A: 1.8-39.2; B: 39.2-76.5; C: 76.6-113.9; D: 113.91-151.3). Patients in the group A and $\mathrm{B}$ had similar prognosis $(p>0.05)$. We then combined the group A and B into A' (0-76.5 points). Patients in group $A^{\prime}$ had a 5-year survival rate of $86.1 \%$, patients in group B'(76.5-113.9 points) had a 5-year survival rate of 59.1\%, whereas patients in group C'(113.9-151.3 points) had a 5 -year survival rate of $28.9 \%(P<0.001)$. Ultimately, the prognostic score was able to stratify patients into 3 distinct prognostic groups (Figure 4).

\section{DISCUSSION}

Liver transplantation provides the best oncological resection and simultaneously solves the underlying liver dysfunction. The Milan criteria based on tumor number and tumor size is widely accepted for liver transplantation [2]. In order to benefit more HCC patients, expansion 
criteria, such as the UCSF, up-to-seven and Hangzhou criteria, were indentified to be applicable and effective [4-6]. Meanwhile, recent studies had demonstrated that patients with HCC beyond the Milan criteria gained more survival benefits from transplantation than surgical resection [3, 7]. Moreover, living donor LT (LDLT) had alleviated the shortage of liver donation without promising outcome compared with donation after cardiac death liver transplantation [17]. There was increasing number of HCC patients beyond the transplant criteria receiving LT. For postoperative individual survival prediction, we created a predictive model. In this current study, we included patients within and beyond transplantation criteria. Patients with major vascular invasion were excluded since advance stage HCC were not recommended for LT in our center. Based on multivariate analysis, tumor size, tumor number and tumor differentiation were included in this model. Tumor size and tumor number were most important indicator of tumor burden. The two factors greatly impacted the prognosis of HCC after LT and surgical resection [18, 19]. Consistent with previously published studies, poor differentiation was related to high incidence of recurrence and poor survival after hepatecomy or after transplantation [20]. Zheng $\mathrm{SS}$, et al. and Cillo $\mathrm{U}$, et al. took tumor differentiation into account for selecting the HCC recipient to achieve satisfactory long-term survival, [5, 21, 22].

Some studies suggested the combination of AFP could increase the accuracy for predictive model [23, 24], while some study did not demonstrated the prognostic value of AFP [4], The role of AFP on patient long-term survival after liver transplantation remains controversial. In the current study, preoperative serum AFP leve was not identified as a predictor in the multivariate model although it was signicficant in the univariate analysis. These retrospective studies and small sample size might give rise to these differences. MVI was an established risk factor in HCC. It has predictive value for HCC patients undergoing hepatetectomy or LDLT [25]. However, some study indentified that it had limited predictive value in the prognosis in transplanted patients including the current

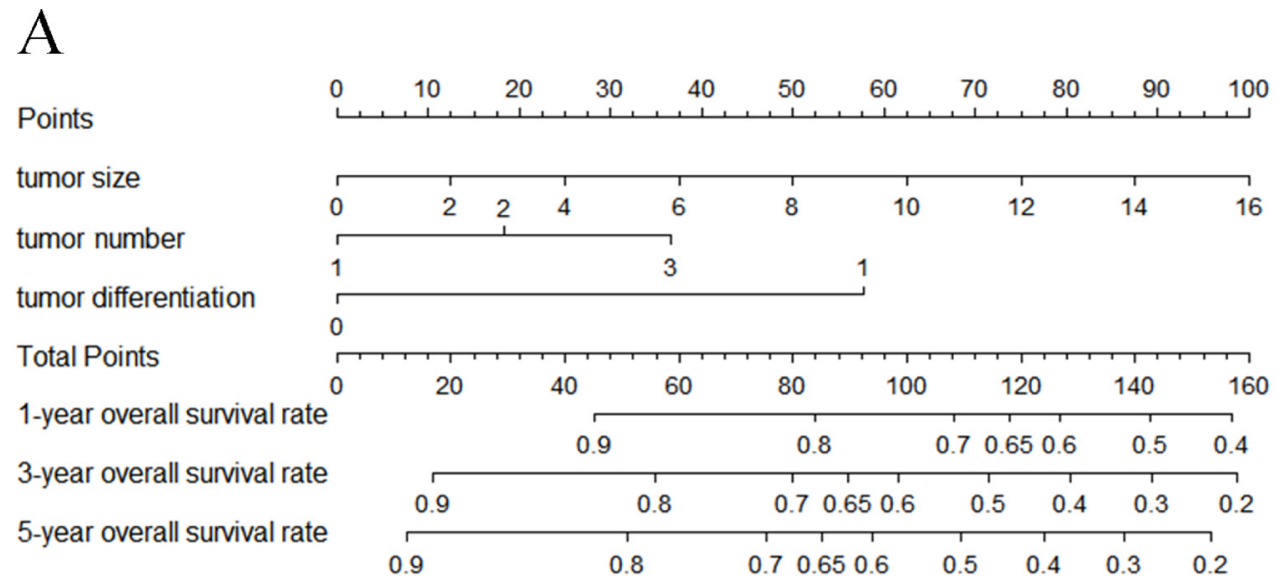

\section{B}

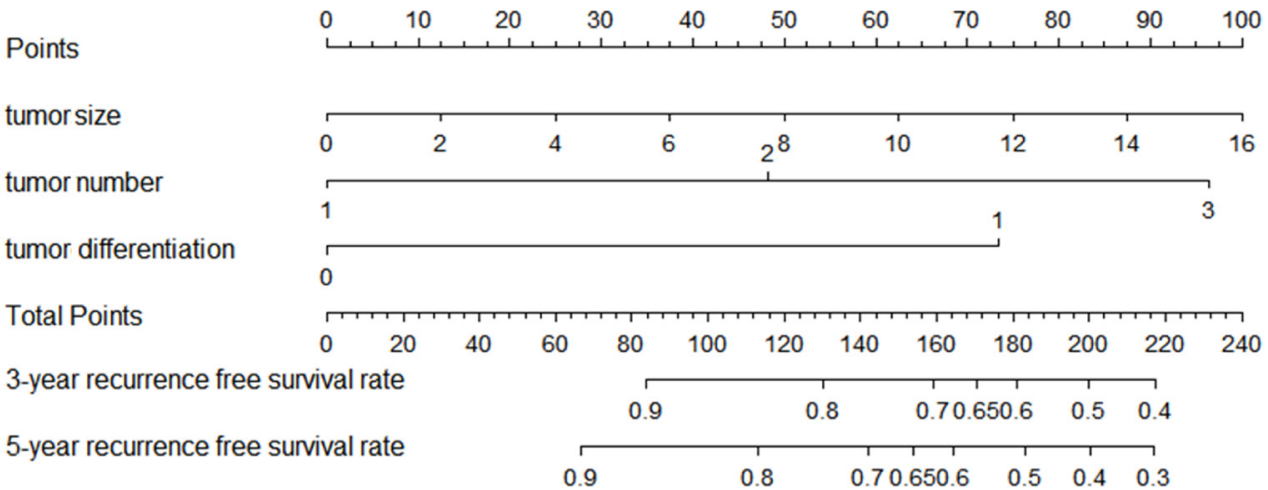

Figure 1: Prognostic nomograms for predicting post-transplantation hepatocellular carcinoma (HCC) overall survival(OS) (A) and recurrence free survival(RFS) (B). For each predictor, a straight upward line is drawn to determine the points. The cumulative points are plotted on the total points bar, and a straight downward line yields the 3-, 5-, and 10-year estimated post-transplantation survival risk. Tumor differentiation: $0=$ moderate-well differentiation; $1=$ poor differentiation. Tumor number: $0=$ one, $1=$ two, $3=$ more. 
study [26]. In our study, we used the status of tumor differentiation to represent the tumor biology instead of MVI [5]. Recently, one multicenter and large sample size study suggested bridging locoregional therapy in HCC patients does not improve post-LT prognosis [27]. Our study was consistent with this result. There was 73 (34.9\%) HCC patients undergoing LDLT. Many studies including our previous study demenstrated there was no statistically difference between the HCC patients after DCD and LDLT [28, 29].

Currently, our nomogram incorporating tumor size, tumor number and tumor differentiation was applied to individual survival prediction. Formerly Sudan, D. et al. constructed a nomogram for hepatocellular carcinoma recurrence after LT with high $\mathrm{C}$ statistic of 0.79 [30]. Feng Shen et al. developed nomograms for prediction of individual survival of patients who recelved salvage LT after hepatectomy with c static of 0.72 and 0.77 [31]. In our study, we exluded HCC patients with major vascluar invasion or patients with recurrent HCC after hepatectomy. We constructed the DFS and OS nomogram using the three simple virables.

Our prognostic nomogram reflected not only tumor morphology but also tumor biology, possibly resulting in better predictive performance. The proposed nomogram demonstrated good discrimination, with a $\mathrm{C}$-index of 0.70 for OS, while the $\mathrm{C}$-index of Milan criteria was C-index 0.62, UCSF criteria was 0.63, up-to-seven
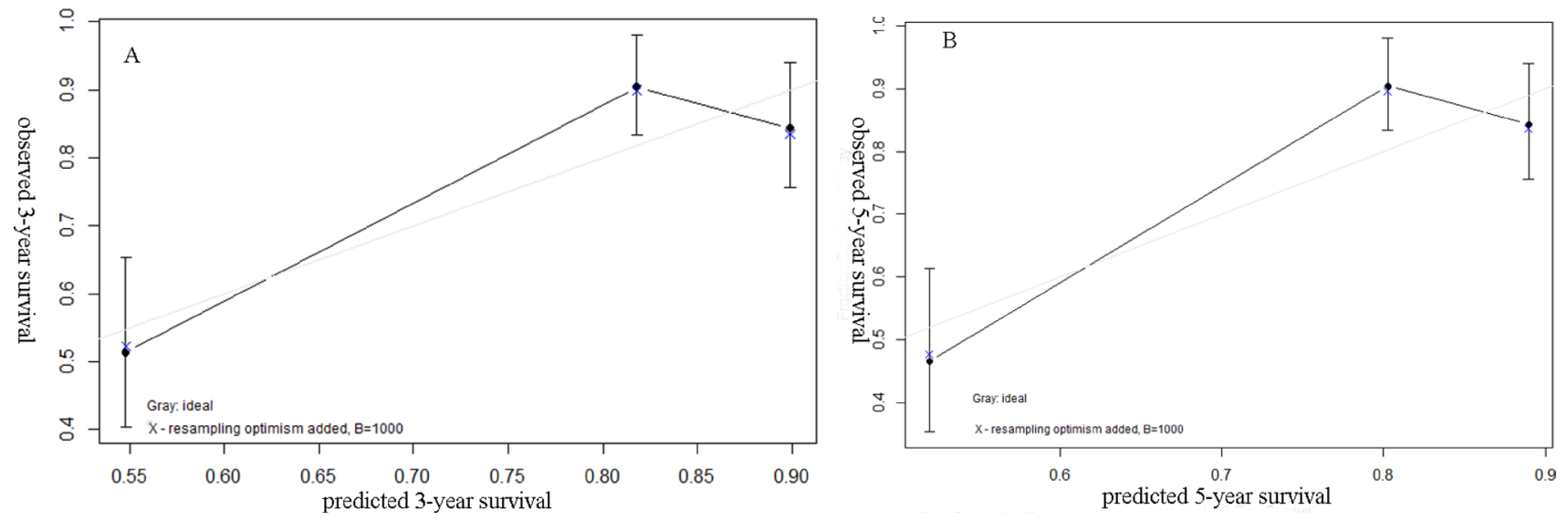

Figure 2: Calibration plot comparing predicted and actual survival probabilities at 3-year (A) or 5-year (B) of follow-up.
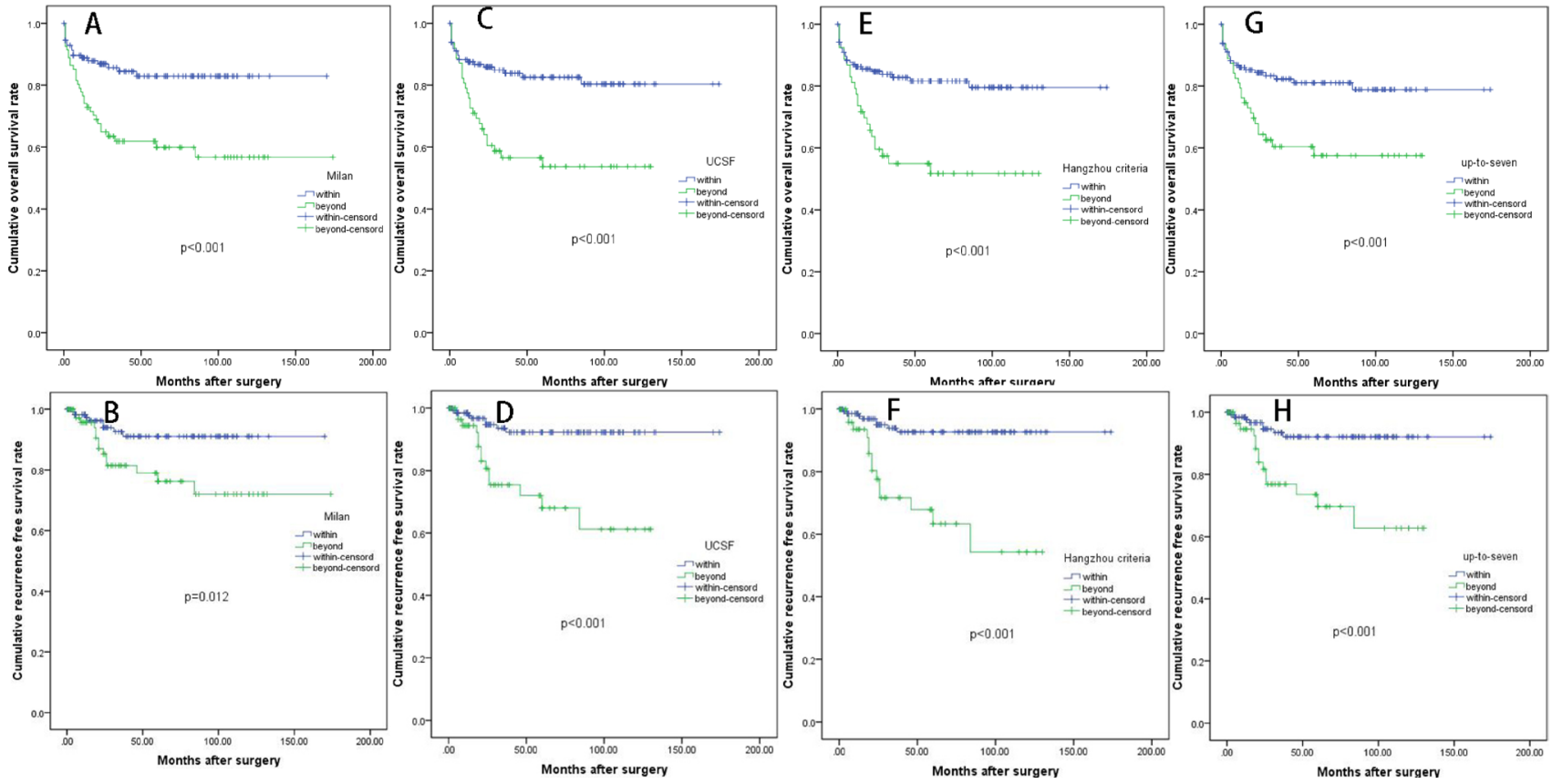

Figure 3: Kaplan-Meier OS and RFS curves of the whole cohort categorized by different liver transplantation criteria: (A and B) the Milan criteria; (C and D) the UCSF criteria; (E and F) the Hangzhou criteria; ( $\mathbf{G}$ and $\mathbf{H})$ the up-to-seven criteria). 
criteria was 0.59 and Hangzhou criteria was 0.61 . These established transplantation criteria were effective and widely acceptable for HCC patient selection. However, for individual survival prediction, they might have some limitations. From the current nomogram, the predicted survival rate was similar to actual survival calculated from the Kaplan-Meier test. Moreover, the proposed nomogram had good internal validation after 1000- bootstrapped resample.
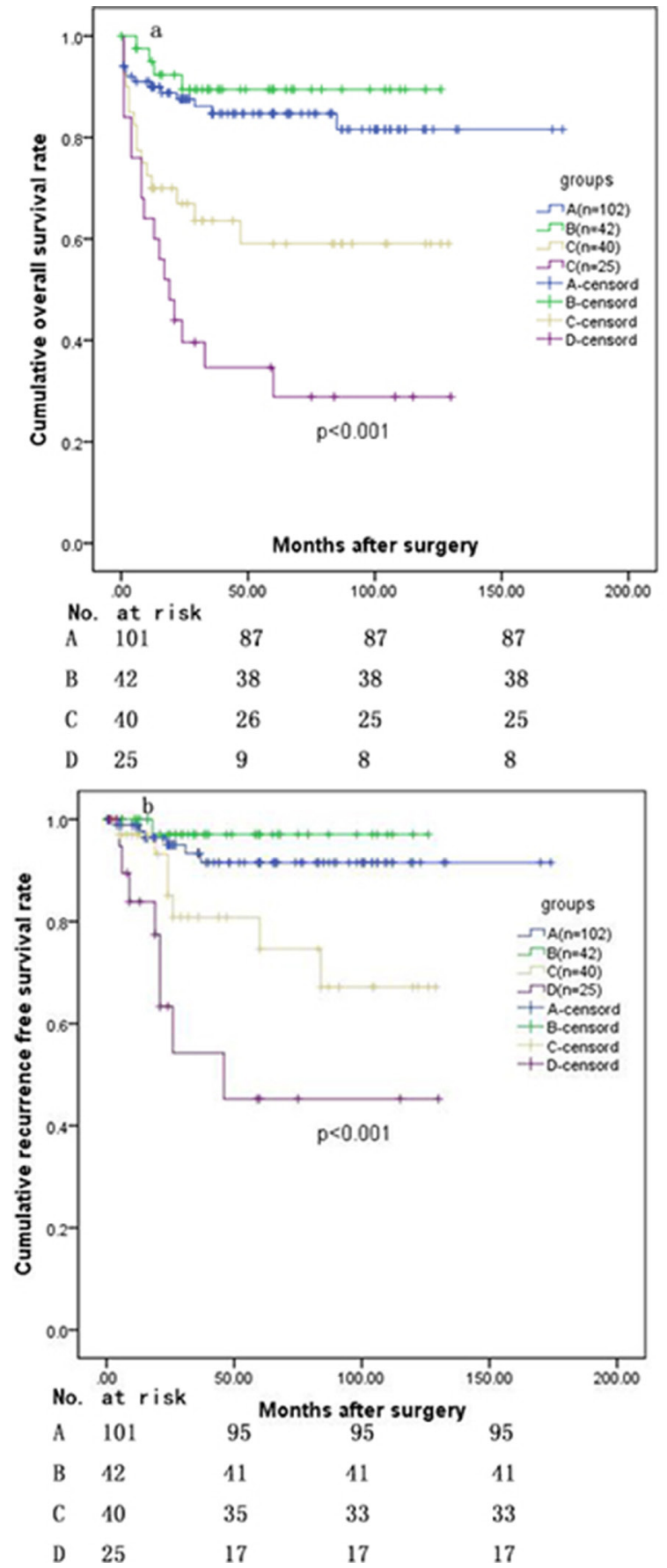

Each patient could gain a score based on the three factors in our model. We stratified all patients into three groups with distinguished prognosis based on the score. Patients in group A' had a 5-year survival rate of $86.1 \%$, patients in group B' had a 5-year survival rate of 59.1\%, whereas patients in group $C^{\prime}$ had a 5-year survival rate of $28.9 \%$. The prognostic score is simple and effective in stratifying the different prognostic factors. Previous studies suggested that 5-year survival rate above 50.0\%
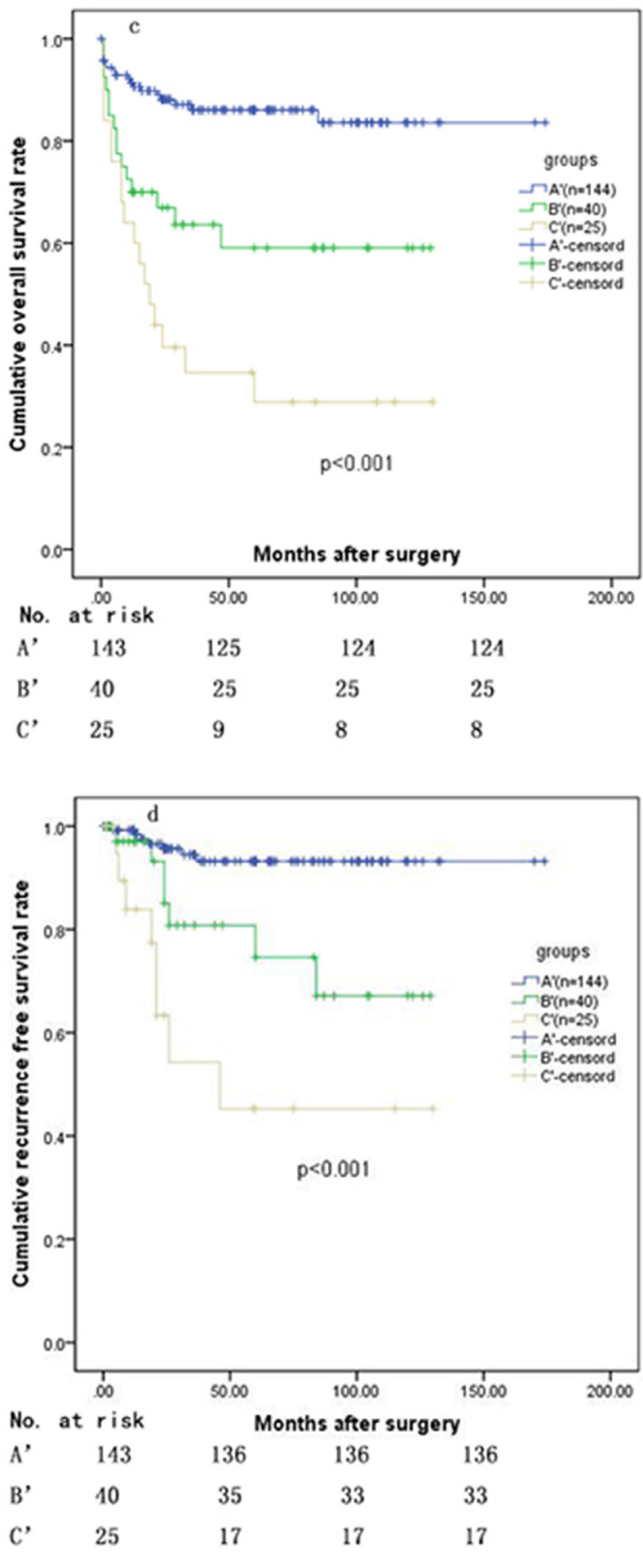

Figure 4: Kaplan-Meier curves demonstrating OS (a) and RFS (b) according to Quartiles of the total points (A: 1.8-39.2 points; B: 39.2-75.5 points; C: 75.6-113.9 points; D: 113.9-151.3 points); (c) OS and (d) RFS after combination of group A and B (A': 1.8-76.5 points; B': 76.6-113.9 points; C': 113.9-151.3 points). 
after LT is acceptable $[8,9]$. From our study, patients with score above 113.9 points had poor prognosis after transplantation. This might aid in deciding organ allocation for liver transplantation if the status of tumor differentiation can be identified by biopsy before LT. Notably, this nomogram was helpful in individualized post-operative survival prediction in clinical practice.

However, the current study had several limitations. Firstly, this was a retrospective study and the sample size was relatively small. The nomogram for recurrent HCC after hepatectomy required to be created in future study. Secondly, this nomogram had good performance for prediction of prognosis but it required external validation. Thirdly, preoperative liver biopsy was needed to indentify tumor differentiation. This might limit the wide application for recipient selection.

\section{MATERIALS AND METHODS}

\section{Patients}

Between Juanuary 2002 and September 2015, 209 qualified HCC patients who received liver transplantation in the West China Hospital were collected for this study. All recipients were followed up until June 2016 or until death. The inclusion criteria: 1) histologically proven to be HCC; 2) without major vascular invasion. The exclusion criteria: 1) concurrent malignancy; 2) initially treated by hepatectomy; 3 ) died within 3 months; 4) follow up for less than 6 months. All liver grafts were voluntarily donated after cardiac death or by living donors. All donations were approved by the West China Hospital Ethics Committee. This retrospective study was conducted in accordance with the Declaration of Helsinki and was approved by the Ethic Committee of West China Hospital.

\section{Post-transplantation immunosuppression and antiviral protocol}

Immunosuppression and antiviral protocol were same as our previous study [15]. The basic regimen of immunosuppressive therapy consisted of corticosteroids, Tacrolimus (TAC) and mycophenolate mofetil (MMF). Methylprednisolone was given intravenously on the first week after transplantation. Intravenous corticosteroid was changed to oral prednisone, which was also withdrawn 3-6 mo after transplantation. The initial dose of TAC was $0.05-0.10 \mathrm{mg} / \mathrm{kg}$ per day and was adjusted according to liver function and TAC serum concentration. MMF was individualized between $1.0 \mathrm{~g} / \mathrm{d}$ and $1.5 \mathrm{~g} / \mathrm{d}$ initially and was discontinued when severe side effects occurred and in long-term survivors with stable graft function after 6 months post LT. After 6 mo post-LT, we reduced the TAC dosage very slowly and carefully while closely monitoring allograft function to maintain TAC concentration as low as possible. Steroid pulse therapy was used after the rejection developed.
For patients with HBV infesction, 4000IU of hepatitis B immune globulin were administrated at the anhepatic phase. Lamivudine was also administrated and hepatitis B immune globulin was regularly administered after surgery.

\section{Follow up}

TAC concentrations, allograft function and renal function were monitored daily during the first week following transplantation, weekly during the first month after LT, monthly within 3 mo and every 3-6 mo thereafter. The patients were followed up for HCC recurrence every 3 mo for the first years, every 3-6 mo thereafter. Alphafetoprotein level (AFP) test and liver ultrasound were performed at each investigation. Suspicious lesions in the liver or lung were confirmed by magnetic resonance imaging (MRI). Resection, radiofrequency ablation (RFA), transcatheter arterial chemoembolization (TACE), systematic therapy and best care support were recommended for HCC recurrence by the Multidisciplinary Team (MDT) based on the transplanted liver, tumor status and general condition of the patients. Overall survival (OS) was defined as time from operation to death or the last date of followup. Recurrence free survival (RFS) was defined as the time from operation to the day of HCC recurrence including intra-hepatic recurrence and/or distant metastases. The primary endpoint was overall survival.

\section{Statistical methods}

Continuous variables were expressed as mean \pm standard deviation and compared by Student's $t$ test or Mann-Whitney $U$ test (non-normal distribution data). Categorical data was shown as frequency and assessed by Fisher's exact test and two-tailed $\chi^{2}$ test. Survival curve was plotted using the Kaplan-Meier method and compared using the log-rank test. Potential risk factors with $P<0.1$ in univariate analysis were included in the Cox proportional hazards model using forward step-wise selection process. The statistical analysis was carried out using the SPSS 20.0.

Independent variables were then incorporated into the nomogram using the package of rms in R. The predictive performance of the nomograms were measured by concordance index (C-index) using the rcorrp.cens package in Hmisc in R and assessed by calibration curve comparing nomogram-predicted versus actually observed Kaplane-Meier estimates of probability [12, 16]. Bootstraps with 1000 resample were used when generating calibration curve. The statistical analysis was carried out using $\mathrm{R}$ software version 3.3.0. All tests were two-sided and a $p$ value $<0.05$ was considered statistically significant.

\section{Author contributions}

Tianfu Wen proposed the study. Junyi Shen and Chuan Li performed the research. Junyi Shen and Jun 
Wen analyzed the data. Junyi Shen and Jun Wen wrote and revised the draft. Tianfu Wen and Highness Tholakkara Nazar reviewed the paper. All authors contributed to the design, interpretation and further drafts of the study.

\section{CONFLICTS OF INTEREST}

none.

\section{FUNDING}

This work was supported by grants from Scientific and Technological Support Project of Sichuan Province (2015SZ0049 and 2016SZ0025) and was supported by "The Fundamental Research Funds for the Central Universities".

\section{REFERENCES}

1. Torre LA, Bray F, Siegel RL, Ferlay J, Lortet-Tieulent J, Jemal A. Global cancer statistics, 2012. CA: A Cancer Journal for Clinicians. 2015; 65:87-108. https://doi. org/10.3322/caac.21262.

2. Mazzaferro V, Regalia E, Doci R, Andreola S, Pulvirenti A, Bozzetti F, Montalto F, Ammatuna M, Morabito A, Gennari L. Liver transplantation for the treatment of small hepatocellular carcinomas in patients with cirrhosis. N Engl J Med. 1996; 334:693-9. https://doi.org/10.1056/ nejm199603143341104.

3. Xu X, Lu D, Ling Q, Wei X, Wu J, Zhou L, Yan S, Wu L, Geng L, Ke Q, Gao F, Tu Z, Wang W, et al. Liver transplantation for hepatocellular carcinoma beyond the Milan criteria. Gut. 2015. https://doi.org/10.1136/ gutjnl-2014-308513.

4. Yao FY, Ferrell L, Bass NM, Watson JJ, Bacchetti P, Venook A, Ascher NL, Roberts JP. Liver transplantation for hepatocellular carcinoma: expansion of the tumor size limits does not adversely impact survival. Hepatology. 2001; 33:1394-403. https://doi.org/10.1053/jhep.2001.24563.

5. Zheng $\mathrm{SS}, \mathrm{Xu} \mathrm{X}, \mathrm{Wu}$ J, Chen J, Wang WL, Zhang M, Liang TB, Wu LM. Liver transplantation for hepatocellular carcinoma: Hangzhou experiences. Transplantation. 2008; 85:1726-32. https://doi.org/10.1097/ TP.0b013e31816b67e4.

6. Mazzaferro V, Llovet JM, Miceli R, Bhoori S, Schiavo M, Mariani L, Camerini T, Roayaie S, Schwartz ME, Grazi GL, Adam R, Neuhaus P, Salizzoni M, et al. Predicting survival after liver transplantation in patients with hepatocellular carcinoma beyond the Milan criteria: a retrospective, exploratory analysis. Lancet Oncol. 2009; 10:35-43. https:// doi.org/10.1016/s1470-2045(08)70284-5.

7. Lu Q, Lu C, Li J, Ling W, Qi X, He D, Liu J, Wen T, Wu $\mathrm{H}$, Zhu H, Luo Y. Stiffness Value and Serum Biomarkers in Liver Fibrosis Staging: Study in Large Surgical Specimens in Patients with Chronic Hepatitis B. Radiology. 2016; 280:290-9. https://doi.org/10.1148/radiol.2016151229.

8. Volk ML, Vijan S, Marrero JA. A novel model measuring the harm of transplanting hepatocellular carcinoma exceeding Milan criteria. Am J Transplant. 2008; 8:839-46. https://doi.org/10.1111/j.1600-6143.2007.02138.x.

9. Bruix J, Fuster J, Llovet JM. Liver transplantation for hepatocellular carcinoma: Foucault pendulum versus evidence-based decision. Liver Transpl. 2003; 9:700-2. https://doi.org/10.1053/jlts.2003.50124.

10. Poon RT, Fan ST, Lo CM, Liu CL, Wong J. Difference in tumor invasiveness in cirrhotic patients with hepatocellular carcinoma fulfilling the Milan criteria treated by resection and transplantation: impact on long-term survival. Ann Surg. 2007; 245:51-8. https://doi.org/10.1097/01. sla.0000225255.01668.65.

11. Lee KK, Kim DG, Moon IS, Lee MD, Park JH. Liver transplantation versus liver resection for the treatment of hepatocellular carcinoma. J Surg Oncol. 2010; 101:47-53. https://doi.org/10.1002/jso.21415.

12. Wang Y, Li J, Xia Y, Gong R, Wang K, Yan Z, Wan X, Liu G, Wu D, Shi L, Lau W, Wu M, Shen F. Prognostic nomogram for intrahepatic cholangiocarcinoma after partial hepatectomy. J Clin Oncol. 2013; 31:1188-95. https://doi. org/10.1200/jco.2012.41.5984.

13. Song KY, Park YG, Jeon HM, Park CH. A nomogram for predicting individual survival of patients with gastric cancer who underwent radical surgery with extended lymph node dissection. Gastric Cancer. 2014; 17:287-93. https://doi. org/10.1007/s10120-013-0270-x.

14. Li Y, Xia Y, Li J, Wu D, Wan X, Wang K, Wu M, Liu J, Lau WY, Shen F. Prognostic Nomograms for Pre- and Postoperative Predictions of Long-Term Survival for Patients Who Underwent Liver Resection for Huge Hepatocellular Carcinoma. J Am Coll Surg. 2015; 221:96274.e4. https://doi.org/10.1016/j.jamcollsurg.2015.08.003.

15. Tanaka S, Kaibori M, Ueno M, Wada H, Hirokawa F, Nakai T, Iida H, Eguchi H, Hayashi M, Kubo S. Surgical Outcomes for the Ruptured Hepatocellular Carcinoma: Multicenter Analysis with a Case-Controlled Study. J Gastrointest Surg. 2016; 20:2021-34. https://doi. org/10.1007/s11605-016-3280-2.

16. Vickers AJ, Kattan MW, Daniel S. Method for evaluating prediction models that apply the results of randomized trials to individual patients. Trials. 2007; 8:14. https://doi. org/10.1186/1745-6215-8-14.

17. Li C, Wen TF, Yan LN, Li B, Yang JY, Wang WT, Xu MQ, Wei YG. Outcome of hepatocellular carcinoma treated by liver transplantation: comparison of living donor and deceased donor transplantation. Hepatobiliary Pancreat Dis Int. 2010; 9:366-9.

18. Wang BL, Tian L, Gao XH, Ma XL, Wu J, Zhang CY, Zhou Y, Guo W, Yang XR. Dynamic change of the systemic immune inflammation index predicts the prognosis of 
patients with hepatocellular carcinoma after curative resection. Clin Chem Lab Med. 2016; 54:1963-9. https:// doi.org/10.1515/cclm-2015-1191.

19. Zhou J, Wang Z, Qiu SJ, Huang XW, Sun J, Gu W, Fan J. Surgical treatment for early hepatocellular carcinoma: comparison of resection and liver transplantation. J Cancer Res Clin Oncol. 2010; 136:1453-60. https://doi. org/10.1007/s00432-010-0802-2.

20. Calderaro J, Rousseau B, Amaddeo G, Mercey M, Charpy C, Costentin C, Luciani A, Zafrani ES, Laurent A, Azoulay D, Lafdil F, Pawlotsky JM. Programmed death ligand 1 expression in hepatocellular carcinoma: Relationship With clinical and pathological features. Hepatology. 2016; 64:2038-46. https://doi.org/10.1002/hep.28710.

21. Xu SL, Zhang YC, Wang GY, Yang Q, Liu B, Zhang J, Li H, Wang GS, Yang Y, Chen GH. Survival analysis of sirolimus-based immunosuppression in liver transplantation in patients with hepatocellular carcinoma. Clin Res Hepatol Gastroenterol. 2016; 40:674-81. https://doi.org/10.1016/j. clinre.2016.03.006.

22. Cillo U, Vitale A, Bassanello M, Boccagni P, Brolese A, Zanus G, Burra P, Fagiuoli S, Farinati F, Rugge M, D'Amico DF. Liver transplantation for the treatment of moderately or well-differentiated hepatocellular carcinoma. Ann Surg. 2004; 239:150-9. https://doi.org/10.1097/01. sla.0000109146.72827.76.

23. Duvoux C, Roudot-Thoraval F, Decaens T, Pessione F, Badran H, Piardi T, Francoz C, Compagnon P, Vanlemmens C, Dumortier J, Dharancy S, Gugenheim J, Bernard PH, et al. Liver transplantation for hepatocellular carcinoma: a model including alpha-fetoprotein improves the performance of Milan criteria. Gastroenterology. 2012; 143:986-94.e3; quiz e14-5. https://doi.org/10.1053/j. gastro.2012.05.052.

24. Grat M, Wronka KM, Stypulkowski J, Bik E, Krasnodebski M, Masior L, Lewandowski Z, Grat K, Patkowski W, Krawczyk M. The Warsaw Proposal for the Use of Extended Selection Criteria in Liver Transplantation for Hepatocellular Cancer. Annals Of Surgical Oncology. 2017; 24:526-34. https://doi.org/10.1245/s10434-016-5500-0.

25. Iguchi T, Shirabe K, Aishima S, Wang HL, Fujita N, Ninomiya $M$, Yamashita $Y$, Ikegami $T$, Uchiyama $H$, Yoshizumi T, Oda Y, Maehara Y. New Pathologic Stratification of Microvascular Invasion in Hepatocellular Carcinoma: Predicting Prognosis After Living-donor Liver Transplantation. Transplantation. 2015; 99:1236-42. https:// doi.org/10.1097/Tp.0000000000000489.
26. Grat M, Stypulkowski J, Patkowski W, Bik E, Krasnodebski M, Wronka KM, Lewandowski Z, Wasilewicz M, Grat K, Masior L, Ligocka J, Krawczyk M. Limitations of predicting microvascular invasion in patients with hepatocellular cancer prior to liver transplantation. Scientific Reports. 2017; 7:39881. https://doi.org/10.1038/Srep39881.

27. Agopian VG, Harlander-Locke MP, Ruiz RM, Klintmalm GB, Senguttuvan S, Florman SS, Haydel B, Hoteit M, Levine MH, Lee DD, Taner CB, Verna EC, Halazun KJ, et al. Impact of Pretransplant Bridging Locoregional Therapy for Patients With Hepatocellular Carcinoma Within Milan Criteria Undergoing Liver Transplantation Analysis of 3601 Patients From the US Multicenter HCC Transplant Consortium. Annals Of Surgery. 2017; 266:525-35. https:// doi.org/10.1097/Sla.0000000000002381.

28. Li C, Zhu WJ, Wen TF, Dai Y, Yan LN, Li B, Yang JY, Wang WT, Xu MQ. Child-Pugh A hepatitis B-related cirrhotic patients with a single hepatocellular carcinoma up to $5 \mathrm{~cm}$ : liver transplantation vs. resection. J Gastrointest Surg. 2014; 18:1469-76. https://doi.org/10.1007/s11605-014-2550-0.

29. Ninomiya M, Shirabe K, Facciuto ME, Schwartz ME, Florman SS, Yoshizumi T, Harimoto N, Ikegami T, Uchiyama H, Maehara Y. Comparative Study of Living and Deceased Donor Liver Transplantation as a Treatment for Hepatocellular Carcinoma. Journal Of the American College Of Surgeons. 2015; 220:297-.94. https://doi. org/10.1016/j.jamcollsurg.2014.12.009.

30. Sudan D, Chapman WC, Cameron JL, Agopian V. A Novel Prognostic Nomogram Accurately Predicts Hepatocellular Carcinoma Recurrence after Liver Transplantation: Analysis of 865 Consecutive Liver Transplant Recipients Discussion. Journal Of the American College Of Surgeons. 2015; 220:427-9.

31. Zou QF, Li J, Wu D, Yan ZL, Wan XY, Wang K, Shi LH, Lau WY, Wu MC, Shen F. Nomograms for Pre-operative and Post-operative Prediction of Long-Term Survival of Patients Who Underwent Repeat Hepatectomy for Recurrent Hepatocellular Carcinoma. Annals Of Surgical Oncology. 2016; 23:2618-26. https://doi.org/10.1245/ s10434-016-5136-0. 